\title{
OS MÉTODOS OUANTITATIVOS DE ANÁLISE DE INVESTIMENTOS
}

\author{
Alexandre Assaf Neto \\ Professor - Doutor do Departamento de \\ Contabilidade e Atuária da FEA/USP.
}

\begin{abstract}
O estudo de avaliação de investimentos se refere basicamente às decisões de aplicações de ca pital em projetos que prometem retornos por vários períodos consecutivos.

O tema se in sere no âmbito $d a$ a dministração financeira em long o prazo, promovendo repercussões importantes sobre o desempenho futuro da empresa e, ainda em termos agregados, sobre o crescimento da
\end{abstract} economia.

Uma empresa, em determinado instante, pode ser vista como um conjunto de projetos de investimento em diferentes momentos de execução. O seu objetivo financeiro, ao avaliar alternativas de investimento, é o de maximizar a contribuição $\mathrm{m}$ arginal desses $r$ ecursos $d$ e capital, pr omovendo $o$ in cremento de su a r iqueza líquida.

É im portante r essaltar q ue o in vestimento de capital se apresenta geralmente como uma parte (algumas $v$ ezes pe quena) $d$ o $p$ rocesso $d$ e $t$ omada de de cisões em presariais. $F$ reqüentemente, objetivos estratégicos se apresentam como fatores decisoriais relevantes na seleção de projetos de investimentos. Esta realidade frusta, em diversos momentos, posições mais teóricas de se id entificar as melhores alternativas a partir unicamente dos métodos quantitativos de avaliação de investimentos. Outros fatores de importância são também considerados na avaliação, permitindo incorporar um estudo de natureza qualitativa.

O segmento de investimento de capital é bastante complexo e amplo, envolvendo inúmeros critérios e métodos de análise. O presente trabalho não tem o intuito de abordar todas as suas partes. $O$ objetivo básico é o de avaliar, dentro de um posicionamento mais crítico, os principais aspectos dos métodos quantitativos mais utilizados pelas empresas para análise de investimentos.

\section{INFORMAÇÕES MÍNIMAS PARA AVALIAÇÃO DE INVESTIMENTOS}

O processo de av aliação $d$ e in vestimentos de manda uma s érie de in formações $f$ inanceiras, enunciadas $s$ egundo diversos critérios. Da $m$ esma $f$ orma, $d$ iferentes estados de $m$ ercado e $d$ a ec onomia interferem nos critérios de análise de investimentos.

As informações mínimas necessárias são descritas, em seus aspectos essenciais, a seguir.

\section{a) Fluxo de Caixa Líquido}

A avaliação de investimento é executada a partir de fluxo líquido de caixa, medido, para cada período do intervalo de tempo, pela diferença entre os fluxos de entrada e os de saída de caixa. Ne stes fluxos são computadas somente o s m ovimentos efeti vos de recursos, co $\mathrm{m} \mathrm{r}$ eflexos financeiros so bre o caixa, desprezando-se $r$ eceitas e despesas de na tureza em inentemente $c$ ontábil (depreciação, amortização, reavaliação patrimonial, entre outros resultados que não são pagos ou recebidos em termos de caixa).

$A$ aná lise de inv estimento é processada co $\mathrm{m}$ bas e em fluxos de $\mathrm{c}$ aixa, se ndo o dimensionamento desses valores considerado como o aspecto mais importante da decisão. A representatividade dos resultados de um investimento é ba stante dependente do rig or e co nfiabilidade com qu e os fluxos de caixa foram estimados.

A decisão de se avaliar projetos de investimento com base nos resultados de caixa, e não a partir do lucro, é de vida a uma necessidade econômica, revelando a ef etiva capacidade da empresa em remunerar o capital aplicado e reinvestir os benefícios gerados.

Caderno de Estudos no06, São Paulo, FIPECAFI - Outubro/1992 


\section{b) Valores Incrementais}

Os fluxos de caixa são computados em seus valores incrementais, ou sejam, pelos fluxos de entrada e saída de c aixa qu e se or iginam da decisão de inv estimento em consideração. Isto equivale a concluir que, inexistindo o investimento, os fluxos de caixa atribuíveis à proposta deixam de existir.

O fluxo de c aixa in cremental a dotado como modelo básico na an álise de investimento apresenta-se genericamente com a seguinte estrutura:

$\mathrm{FC}=[\Delta \mathrm{ROP}-\mathrm{IR}(\Delta \mathrm{ROP})]+\Delta \mathrm{DND}$

$\mathrm{FC}=\Delta \mathrm{ROP} \times(1-\mathrm{IR})+\Delta \mathrm{DND}$

onde:

$\Delta \mathrm{FC}=$ Fluxo de caixa incremental;

$\Delta \mathrm{ROP}=$ resultado operacional incremental;

$\mathrm{IR}$ = alíquota de imposto de renda aplicável sobre o resultado operacional incremental;

$\Delta$ DND = despesas não desembolsáveis incrementais (depreciação, basicamente)

\section{c) Taxa Mínima de Atratividade}

Na seleção de investimento é necessária a definição prévia da taxa de retorno exigida, isto é, a taxa de atratividade econômica do projeto.

Ao se trabalhar com métodos de fluxo de caixa descontado, a taxa de atratividade constitui-se no parâmetro de avaliação dos projetos, a meta econômica mínima a ser alcançada.

No método do valor presente liquido, a taxa de atratividade é o percentual de desconto dos fluxos de caixa. Sendo o valor presente das entradas menos o das saídas de caixa positivo, há indicação técnica de aceitação do investimento. Em caso contrário, deve ser rejeitado.

No método da taxa interna de retorno, a taxa de atratividade é comparada com o retorno calculado, indicando aceitação quando esta última for, pelo menos, igual à taxa de desconto utilizada.

\section{4) Outras Informações}

Outras informações a respeito do processo de investimento devem ser levadas em consideração na análise.

* Origens das Propostas: expansão, lançamento de novos produtos, modernização, instalação, relocalização.

${ }^{*} \mathrm{~T}$ inos de Investimentos: independentes, economicamente dependentes, mutuamente excludentes, restrições orçamentárias.

É preciso levar em conta, ainda, os aspectos de risco inerentes a todo projeto. Como os investimentos são decisões tomadas fundamentalmente em relação ao futuro, é sempre necessário que se inclua unia avaliação do risco no estudo da viabilidade econômica.

Por exemplo, os fluxos de caixa definidos para as decisões de investimento são valores previstos de ocorrer ao longo de determinado período de tempo futuro, estando associados, evidentemente, às incertezas inerentes às previsões.

Existem diversos métodos de Matemática e Estatística us ada para se a valiar o $r$ isco de um investimento, buscando todos eles conhecer a probabilidade de ocorrência de determinado estado de natureza e seus resultados. Algumas técnicas e métodos bastante ad otados são: Medidas Estatísticas de Dis persão, Distribuição de $\mathrm{P}$ robabilidades, Mé todo $\mathrm{d}$ e $\mathrm{M}$ onte Carlo, Árvores de $\mathrm{D}$ ecisão ( adotada e $\mathrm{m}$ decisões 
seqüenciais), Simulação etc.

Muitas vezes, a unidade decisorial ado ta, de $m$ aneira mais $s$ implificada, o inc remento da taxa de retorno exigida do investimento como critério de avaliação de risco. Dependendo de certas circunstâncias, esta medida nem sempre é adequada.

A moderna teoria de $\mathrm{F}$ inanças ve $\mathrm{m}$ inc orporando, nas dec isões de investimento em co ndições de risco, o método do CAPM - "Capital Asset Pricing Model", ou Modelo de Precificacão de Ativos. O CAPM, em essência, define a remuneração pelo risco através da taxa adotada pelo mercado.

Tratando-se principalmente da economia brasileira, é necessário incluir-se a inflação nas decisões de investimento como um fator de maior risco. Os problemas mais relevantes da inflação são a dificuldade e m prever os seus valores e maior complexidade em considerá-la tecnicamente no processo de análise.

Dentro da influência da inflação sobre a análise de investimento, a sua inclusão é tratada na definição da taxa de desconto a ser utilizada na avaliação dos benefícios e, mais intensamente, sobre os resultados de caixa gerados no tempo.

Diante d o ex posto, os f luxos d e c aixa da s a Iternativas de investimento podem se apresentar expressos sob diferentes formas: ocorrência;

Fluxos de Ca ixa Nominais - en contram-se ex pressos e m valores c orrentes d a ép oca de sua

Fluxos de Caixa Co nstantes - os v alores apresentam o m esmo poder de compra, o u se ja, estão referenciados em moeda de mesma capacidade aquisitiva;

Fluxos de C aixa De scontados - Os valores enc ontram-se todos at ualizados par a a da ta presente através de uma taxa de desconto definida para o investimento.

\section{MÉTODOS DE ANÁLISE}

São definidos a seguir os métodos quantitativos de análise de investimento mais utilizados.

\section{Valor Presente Líuido (NPV)}

Reflete a riqueza em valores absolutos do investimento medida pela diferença en tre o valor presente das entradas de caixa e o valor presente das saídas de caixa, isto é:

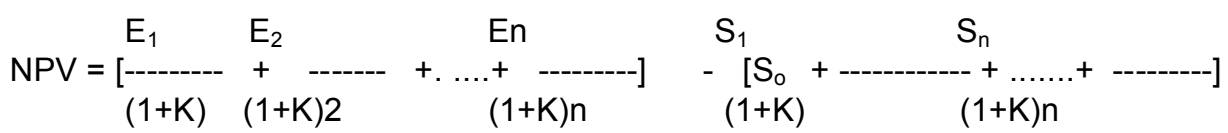

Conseqüentemente:

\begin{tabular}{|c|c|c|c|}
\hline$N \quad E j$ & & $\mathrm{n}$ & Sj \\
\hline$N P V=\left[\begin{array}{ll}\sum_{J=1} & (1+K)\end{array}\right]$ & [ So & $+\sum_{j=1}$ & $(1+K) \mathrm{j}$ \\
\hline
\end{tabular}

Onde:

$E=$ Fluxos esperados de entrada de caixa, ou seja, fluxos operacionais líquidos de caixa gerados pelo investimento;

S = Fluxos de saída de caixa (investimento);

$\mathrm{K}=\quad$ taxa de atratividade do investimento usada para atualizar o fluxo de caixa.

O método do NPV exige a definição prévia desta taxa para descontar os fluxos de caixa.

O NPV, desta forma, é determinado de scontando-se os fluxos financeiros pe la taxa de at ratividade Caderno de Estudos n06, São Paulo, FIPECAFI - Outubro/1992 
(taxa de retorno exigida) definida para o projeto, apurando-se assim o retorno econômico esperado.

$\mathrm{O}$ cr itério de ac eitação-rejeição do método é bastante sim ples: é c onsiderado at raente t odo investimento que apresente um IPV maior o u igual a zero. P rojetos co $\mathrm{m}$ NP V ne gativo ind icam um retorno inferior à taxa mínima requerida, revelando ser economicamente desinteressante sua aceitação.

\section{Taxa Interna de Retorno (IRR)}

A taxa in terna de retorno (IRR) representa a taxa de des conto (taxa de juros) que iguala, num único momento, os fluxos de entrada com os de saída de caixa. Em outras palavras, é a taxa de juros que produz um NPV $=0$.

Genericamente, a IRR é representada, su pondo a at ualização de todos os valores de ca ixa pa ra o momento zero, da forma seguinte:

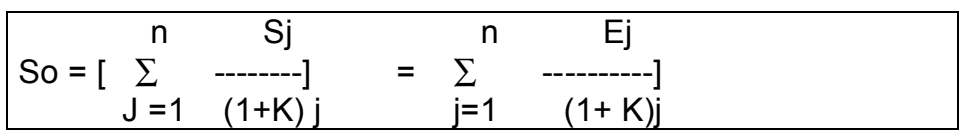

Onde:

1 = taxa de rentabilidade equivalente periódica (IRR).

Pelo en unciado, para o cálculo da taxa interna de retorno é necessário o conhecimento do dis pêndio de capital (ou dispêndios, caso o investimento esteja prevendo mais de uma aplicação de capital) e dos fluxos líquidos de caixa gerados exclusivamente pela decisão.

A IRR reflete a rentabilidade relativa (percentual) de um projeto de investimento expressa em termos de uma taxa de juros equivalente periódica.

A ac eitação ou rejeição do investimento com base neste método é definida pela comparação que se faz entre a IRR encontrada e a taxa de atratividade exigida pela empresa. Se a IRR exceder a taxa mínima de atratividade $\mathrm{o}$ in vestimento é c lassificado como economicamente atraente. Caso contrário, há recomendação técnica de rejeição.

\section{Índice de Lucratividade (IL) e Taxa de Rentabilidade (TR)}

O índice de luc ratividade (IL) é m edido pe la relação entre o valor atualizado dos fluxos operacionais líquidos de entrada de caixa e os de saída de caixa (investimentos), ou seja:

\begin{tabular}{|ccc|}
\hline$\sum^{n}$ & $E j /$ & $(1+K) j$ \\
\hline So $+\sum_{(j=1)}^{n}$ & Sj/ & $(1+K) j$ \\
\hline
\end{tabular}

Indica, para cada $\$ 1$ aplicado em d eterminado inv estimento, qu anto a em presa a purou de retorno, expressos todos os resultados em valores atualizados pela taxa mínima de atratividade.

Quando o índice de lucratividade for superior a 1, 0, in dica um valor presente liquido maior que zero, revelando ser o projeto economicamente atraente. Em caso contrário, IL menor que 1,0, tem-se um indicativo de desinteresse pela alternativa, a qual produz um valor atualizado de en trada de caixa menor que o de saída (NPV negativo).

Por outro lado, a taxa de rentabilidade (TR) consiste na relação entre o NPV, determinado a partir da taxa de atratividade, e o valor atualizado dos dispêndios de capital.

Genericamente, tem-se: 


\begin{tabular}{|c|c|c|c|}
\hline & & JV & \\
\hline & $\sum_{(j=1)}$ & Sj/ & $(1+K) j$ \\
\hline
\end{tabular}
custo/beneficio.

Os dois métodos são representativamente bastante próximos, sendo conhecidos também por relação

\section{Período de "Payback"}

Apesar de não ser um método baseado no critério d e fluxo de c aixa descontado, o p eríodo de "Payback" se destaca pela sua simplicidade e ampla utilização pelas unidades decisórias.

O método consiste, em essência, no cálculo do prazo necessário para que o montante do dispêndio de capital efetuado seja recuperado através dos fluxos líquidos de caixa gerados pelo investimento.

\section{O "payback" convive com duas deficiências:}

a) não leva em conta os fluxos de caixa que ocorrem após o período de "payback";

b) não le va em co nta as magnitudes dos fluxos de ca ixa e s ua distribuição nos períodos que antecedem ao período de "payback".

Diante d essas r estrições, é r ecomendado que o per íodo de "payback" s eja det erminado através do critério de fluxo de caixa descontado, sendo a dimensão do "payback" o tempo gasto para que o NPV passe de negativo para positivo.

No processo de de cisão, o período de "payback" é co mparado com o pa drão es tabelecido pela empresa. Excedendo ao limite fixado, o investimento apresenta indicações de rejeição. A aceitação se revela quando o "payback" for inferior ao padrão.

\section{ANÁLISE COMPARATIVA DOS MÉTODOS PARA UM ÚNICO INVESTIMENTO}

Um projeto de in vestimento tratado in dividualmente é classificado como at raente se ap resentar NPV positivo, ou IRR superior (ou igual) à taxa mínima de retorno requerida, ou um IL maior que 1, O (ou ainda uma TR positiva).

Para um único projeto de investimento, ou para projetos independentes (que podem ser implementados ao mesmo tempo), os métodos de análise que levam em conta os fluxos de caixa descontados convergem sempre para a mesma decisão.

Ilustrativamente, admita o seguinte investimento:

\begin{tabular}{|c|c|c|c|c|c|}
\hline \multicolumn{6}{|c|}{ PERÍODOS } \\
\hline $\mathrm{O}$ & 1 & 234 & & & 5 \\
\hline$-\$ 1200.00$ & \$200 & $\$ 40000$ & $\$ 40000$ & $\$ 60000$ & $\$ 60000$ \\
\hline
\end{tabular}

Definindo-se em 15\% a taxa periódica de atratividade para o investimento, têm-se os seguintes resultados dos métodos de avaliação:

Caderno de Estudos no06, São Paulo, FIPECAFI - Outubro/1992 


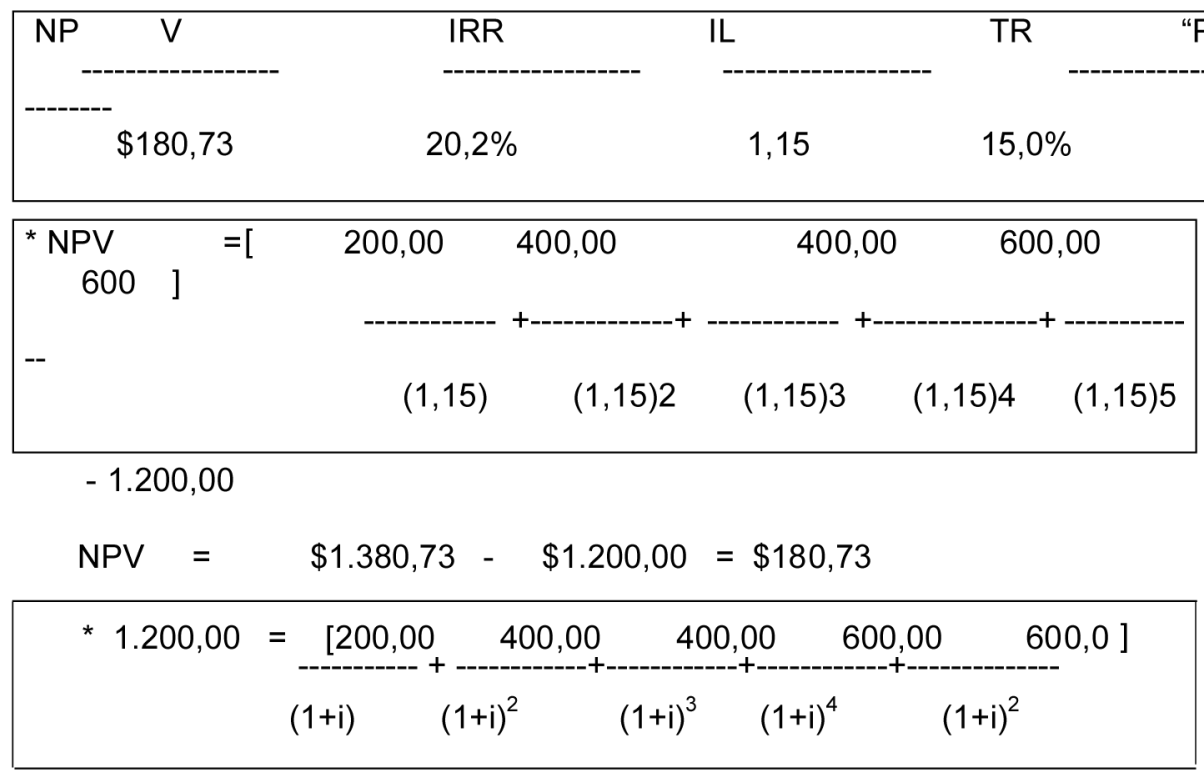

$\operatorname{IRR}(i)=20,2 \%$ ao período

* $\quad \mathrm{IL}=\$ 1.380,73 / \$ 1.200,00=1,15$

* $\quad$ TR $=\$ 180,73 / \$ 1.200,00=15,0 \%$

* $\quad$ "Payback" $=(\$ 1.200,00 / \$ 1.380,73) \times 5$ anos $=4,4$ anos.

Pelos métodos en volvendo fluxos de c aixa descontados, o projeto é classificado como atraente por todos. A presenta um N PV p ositivo, ind icando um r etorno em ex cesso e m r elação a o ga nho ex igido. A IRR supera a taxa de atratividade definida, revelando uma rentabilidade acima da mínima desejada. O IL é maior que 1,0, que representa o ponto de corte entre aceitação-rejeição deste método. Um IL maior 1,0 corrobora, conforme c omentou-se, os r esultados p ositivos dem onstrados pe lo N PV e I RR. Em c onseqüência, a TR é também positiva, atingindo a $15,0 \%$.

Finalmente, o p eríodo de "payback", $r$ evela, e $\mathrm{m}$ valores at ualizados, um a expectativa de $r$ etorno financeiro de aproximadamente 4,4 anos. Este resultado deve ser comparado com o limite-padrão fixado pela empresa.

Desta maneira, trabalhando-se com um único projeto de in vestimento, a ap licação dos métodos de avaliação é processada de maneira bastante simples tendo como característica a total coincidência em termos de decisão.

Os resultados do NPV e IRR podem ser graficamente representados pela Figura 1 abaixo.

Figura 1 - Desempenho Comparativo: NPV x IRR

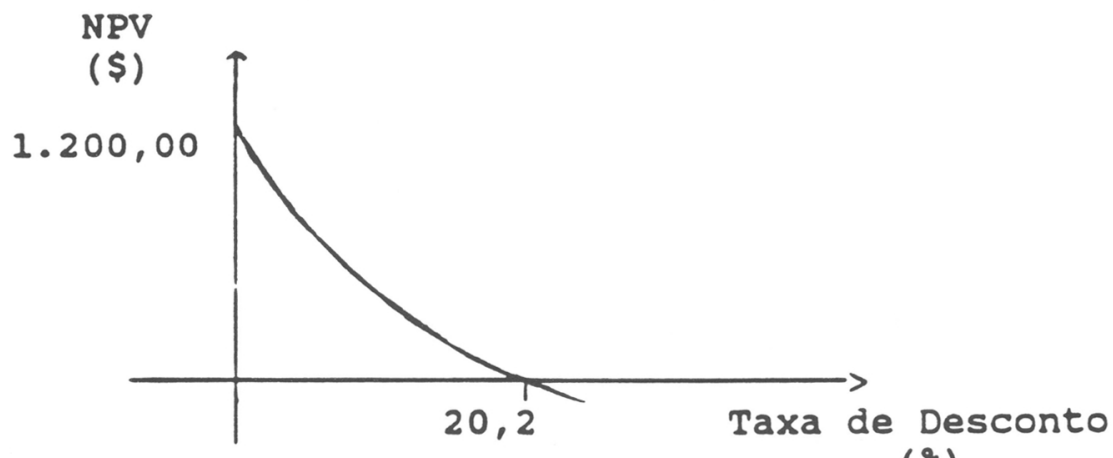

(z)

Caderno de Estudos no06, São Paulo, FIPECAFI - Outubro/1992 
O gráfico c omparativo do NP Ve IRR per mite representar o perfil do inv estimento e su as características de retorno. $\mathrm{O}$ eixo horizontal in corpora diferentes taxas de desconto a s erem a plicadas a os fluxos de caixa. O e ixo ve rtical de termina o $v$ alor p resente lí quido ob tido a pa rtir de ca da taxa de $d$ esconto descrita. O NPV é apurado para um intervalo de taxas razoavelmente amplo, permitindo refletir uma linha de comportamento bastante representativa.

Observe que o NPV decresce á medida que o percentual de desconto se eleva. A linha do NPV corta o eixo horizontal à taxa de $20,2 \%$, ponto em que o NPV é nulo, indicando ser esta a IRR do investimento. Taxas de desconto acima deste valor produzem NPV negativos, indicando o desinteresse pela alternativa.

Na figura 2 processa-se uma comparação entre o valor presente liquido e o período de "payback".

\section{Figura 2 - Perfil do NPV e "Payback"}

\section{NPV}

$1.200,00$

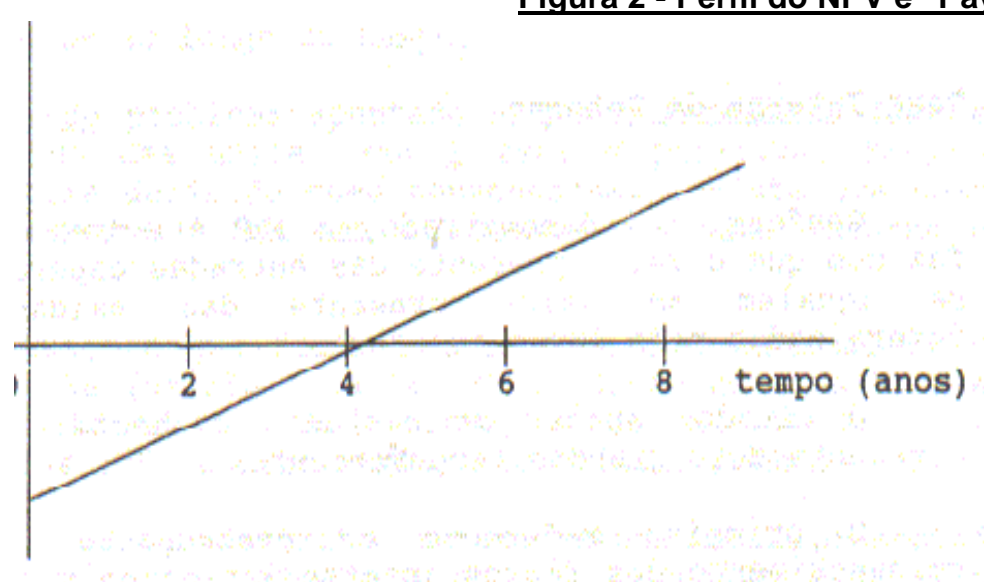

No momento inicial $\left(t_{0}\right)$ o NPV é negativo e igual ao valor do investimento de $\$ 1.200,00$. O NPV vai se incrementando ao longo do tempo de conformidade com a execução dos benefícios de caixa do investimento.

Para a recuperação total do investimento, indicado pela presença de um NPV nulo, são necessários, conforme calculado anteriormente, 4,4 anos. Esta marca temporal é indicada no gráfico a través do ponto de interseção entre a linha do "payback" e o eixo horizontal do tempo.

Por ou tro la do, a o se analisar comparativamente do is ou mais projetos de investimento, os métodos quantitativos $\mathrm{p}$ odem ser co nflitantes em termos de decisão, recomendando algu ns a ac eitação e outros a rejeição de um mesmo investimento. É pe rfeitamente possível, em determinadas cir cunstâncias, deparar-se com situações em que o NPV classifica um determinado investimento $\underline{X}$ como o mais atraente; a IRR indica 0 desinteresse econômico dessa alternativa e seleciona o investimento $Y$ como o que deve ser aceito; a TR, por sua vez, demonstra maior interesse pelo investimento $Z$, e assim por diante.

Nestas condições, a decisão ótima fica bastante prejudicada, impedindo que se alcance o objetivo de maximização da riqueza segundo um único critério.

Os itens seguintes deste trabalho abordam uma análise comparativa dos métodos quantitativos de avaliação de investimentos voltados para a obtenção de uma única decisão ótima.

Caderno de Estudos no06, São Paulo, FIPECAFI - Outubro/1992 


\section{ANÁLISE CRÍTICA}

\section{Taxa Interna de Retorno}

Conforme foi demonstrado, a IRR é a taxa de juros que faz com que o valor presente das entradas esperadas de caixa se iguale ao valor presente das saídas de caixa determinadas pelo investimento.

O método, apesar de bastante conhecido e intuitivo, apresenta dois grandes inconvenientes.

O primeiro refere-se ao pressuposto implícito de reinvestimento dos fluxos intermediários de caixa à própria taxa interna i encontrada. Este pressuposto apresenta-se de difícil execução prática, somente verificada em algumas situações especiais.

Por exemplo, se uma empresa fizer um desembolso imediato de $\$ 3.000,00$ na expectativa de receber $\$ 700,00$ ao final de um ano, $\$ 900,00$ em dois anos, $\$ 1.400,00$ em três anos e $\$ 1.700,00$ em quatro anos, a taxa de retorno (IRR) esperada deste investimento atinge a $17,5 \%$ ao ano.

Esta taxa, no entanto, apesar de corretamente calculada, somente é verdadeira ao se admitir que todos os fluxos de caixa determinados pelo investimento sejam automaticamente reinvestidos à própria IRR de $17,5 \%$ ao ano até o final da vida do projeto.

Assim, ao se reinvestir os fluxos de entradas de caixa (benefícios de caixa) a IRR de $17,5 \%$ apura-se o montante de $\$ 5.723,12$ ao final do $4^{\circ}$. ano, o qual, confrontado geometricamente com o investimento de $\$ 3.000,00$ no momento inicial, revela uma taxa equivalente anual de $17,5 \%$.

No entanto, ao se admitir o reinvestimento destes fluxos de entrada de caixa à taxa de $10 \%$ ao ano, por exemplo, chaga-se a um montante de $\$ 5.260,70$ ao final do $4^{\circ}$. ano, apurando-se uma taxa equivalente composta de $15,0 \%$ ao ano.

Logo, verifica-se que a IRR somente faz sentido se os fluxos de caixa puderem ser automaticamente reaplicados à própria taxa interna de juros calculada. Reinvestimento à taxa de juros inferiores, situações geralmente verificadas na prática, promovem uma redução da IRR calculada.

A taxa interna de retorno, quando usada para interpretar a taxa de retorno de um investimento, é entendida como dependente unicamente dos fluxos de caixa da alternativa de investimento em consideração, não levando em conta as suposições com relação às taxas de reinvestimento destes valores ao longo do tempo.

O segundo problema apontado é que o método da IRR nem sempre produz uma única taxa i real e positiva. Para um fluxo de caixa definido como convencional (existe uma única inversão de sinal) há uma e somente uma taxa interna de retorno.

$\mathrm{E}$, situações que fogem a estrutura convencional de um fluxo de caixa (existe mais de uma inversão de sinal), além de uma só taxa interna de retorno, podem existir situações com múltiplas IRR ou, até mesmo, não existir nenhuma IRR.

A ilustração descrita anteriormente e representada através do diagrama do fluxo de caixa abaixo é do tipo convencional, indicando que a IRR existe e é única.
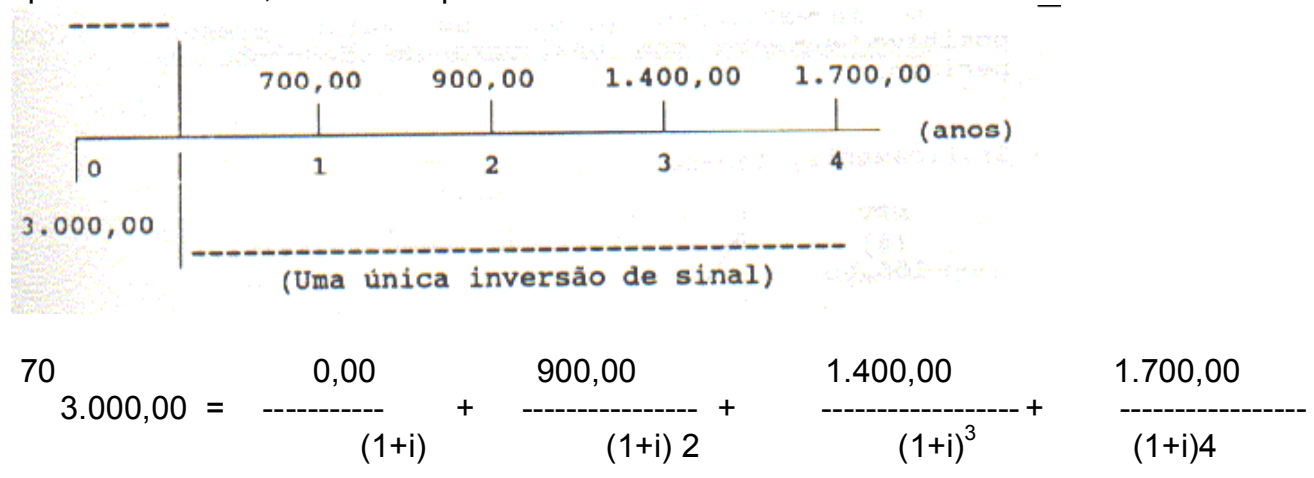

Resolvendo-se:

$I(I R R)=17,5 \%$ ao ano

Caderno de Estudos no06, São Paulo, FIPECAFI - Outubro/1992 
Os exemplos anunciados a seguir visam ilustrar numericamente os fluxos de caixa não—convencionais e seus possíveis resultados pelo uso do método da IRR.

\section{Investimento Não-Convencional Com Uma Única IRR}

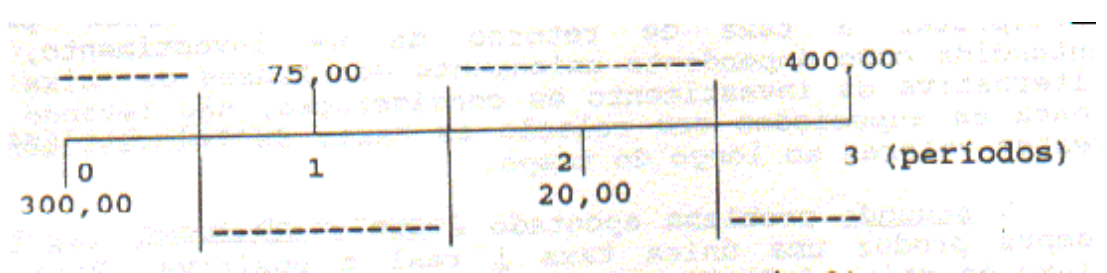

(Mais de Uma Inversão de Sinal)

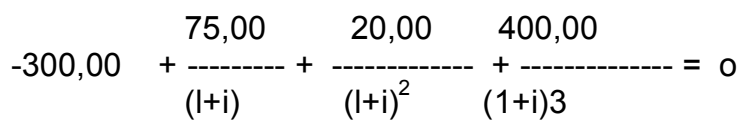

Resolvendo-se:

I $(\mathrm{IRR})=16,9 \%$ ao período

O investimento produz um valor presente líquido positivo somente com uma taxa de desconto de $16,9 \%$ ao período.

Graficamente, tem-se:

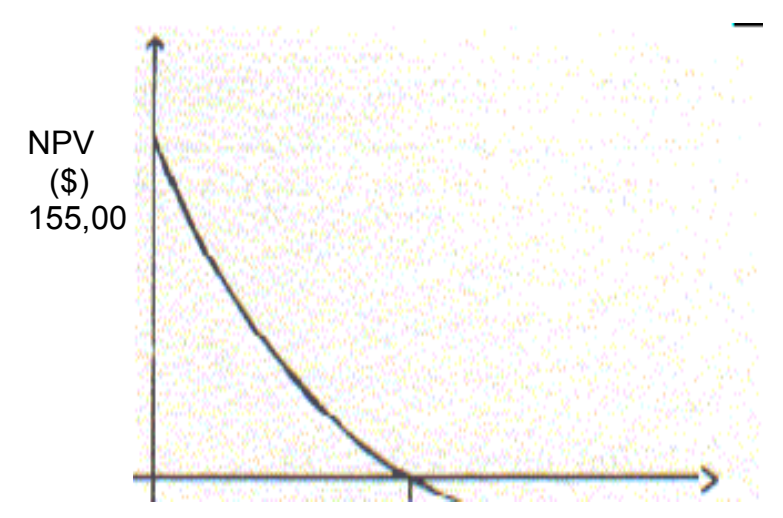

16,9 Taxa de Desconto (\%)

- Investimento Não-Convencional com Mú1tip1as IRR

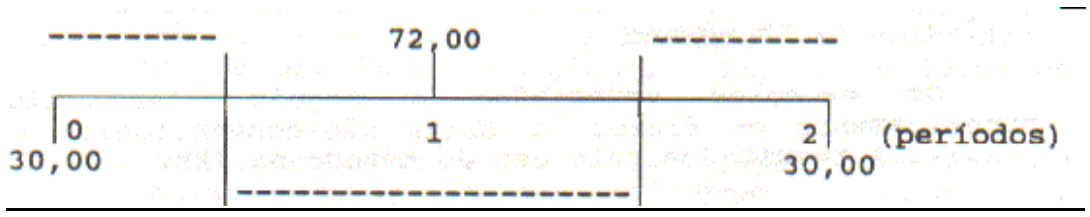

Caderno de Estudos no06, São Paulo, FIPECAFI - Outubro/1992 
Os Métodos Quantitativos de Análise de Investimentos 10

(Mais de Uma Inversão de Sinal)

$-30,00=+72,00 \quad 30,00$

$(I+i) \quad(I+i)^{2}$

$I R R_{1}=+86,33 \%$ ao período

$I R R_{2}=-46,33 \%$ ao período

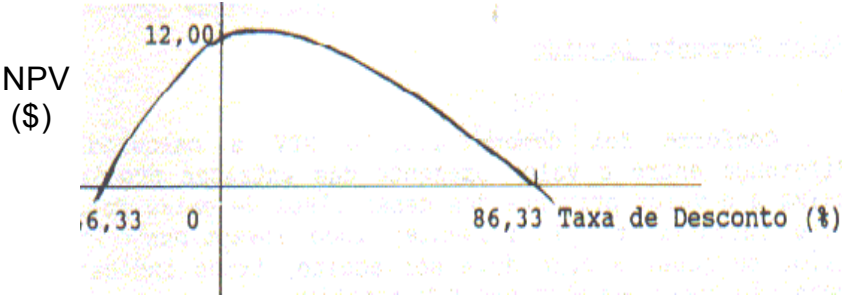

O valor presente líquido se anula com mais de uma taxa de juros, indicando a existência de múltiplas

IRR.

\section{Investimento Não-Convencional com IRR Indeterminada}

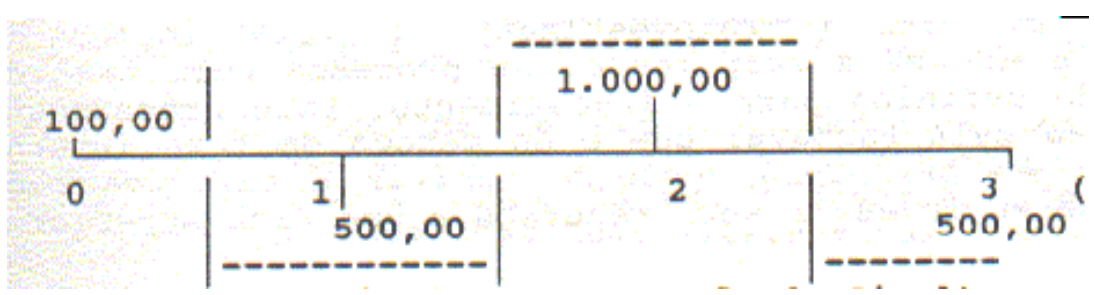

(mais de Uma Inversão de Sinal)

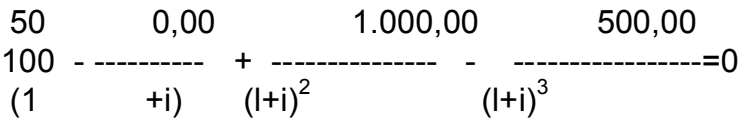

$\mathrm{i}(\mathrm{IRR})=$ indeterminado (não há solução).

A linh a do NP V nã o c orta o e ixo hor izontal e m ne nhuma parte, ap resentando-se sempre maior que zero qualquer que seja a taxa de desconto utilizada.

Existem diversos critérios que objetivam interpretar a existência de múltiplas taxas de retorno. Apesar do fundamento lógico que costumam a presentar, o s m étodos nã o c onseguem pr oporcionar $r$ esultados irrefutáveis. N estas condições, é proposto qu e o m étodo da I RR seja válido so mente pa ra investimentos convencionais, ou seja, para aqueles que apresentam uma única inversão de sinal em seus fluxos de caixa.

Ao se tratar de fluxos de c aixa não-convencionais, s ugere-se a u tilização de ou tros métodos de análise de investimentos, notadamente o valor presente líquido. 
Os Métodos Quantitativos de Análise de Investimentos 11

\section{Valor Presente Líquido}

Conforme foi demonstrado, o N PV é calculado pela dif erença en tre o valor presente das en tradas previstas de caixa e o v alor presente dos desembolsos de caixa requeridos pelo investimento. Basicamente, todo investimento com NPV maior ou igual a zero deve ser aceito, tendo indicativo de rejeição todos aqueles com NPV negativo.

Para uma determinada alternativa de investimento, o NPV indica a riqueza em excesso aos dispêndios determinados pelo projeto.

Identicamente ao método da IRR, o NP V adm ite o pressuposto do $r$ einvestimento a utomático do $s$ fluxos de caixa. A diferença básica, no entanto, é que no método da IRR está implícito o reinvestimento à taxa interna i calculada, e no NPV a replicação se processa pela taxa de atratividade definida para o investimento. Este pressuposto do NPV é bem mais razoável que o d o método da taxa int erna de $r$ etorno; em p rincípio, pode-se admitir a capacidade de toda empresa em investir seus recursos a uma taxa superior à de mercado.

Uma dificuldade notada na ap licação do método do valor presente liquido é que seu significado nem sempre é c orretamente c ompreendido pe la unid ade dec isória. O entendimento da taxa interna de retorno, expressa em porcentagem, é bem mais intuitiva ao analista, facilitando a classificação dos investimentos.

Uma outra a rgumentação da dificuldade do NP V ser mais difundido co mo m étodo de an álise de investimento, é a necessidade de determinação prévia de uma taxa de desconto (Taxa de Atratividade). Isto é ilusório, pois a IRR precisa identicamente ser comparada com a taxa de retorno exigida pelo investimento.

Em su ma, o $\mathrm{m}$ étodo do $\mathrm{v}$ alor pr esente liqu ido é admitido como o $\mathrm{m}$ ais seguro e adequado tecnicamente em relação à IRR. É m encionado que a riqueza de u m inv estimento é m elhor mensurada em valores absolutos, e não em valores relativos.

\section{Extensões ao Perfil dos Métodos do NPV e IRR}

Com o intuito de melhor compreender-se a relação en tre o NPV e a IRR é interessante descrever-se graficamente os resultados dos fluxos de caixa de um investimento interpretado sob dois ângulos: aplicação de capital e tomada de capital emprestado.
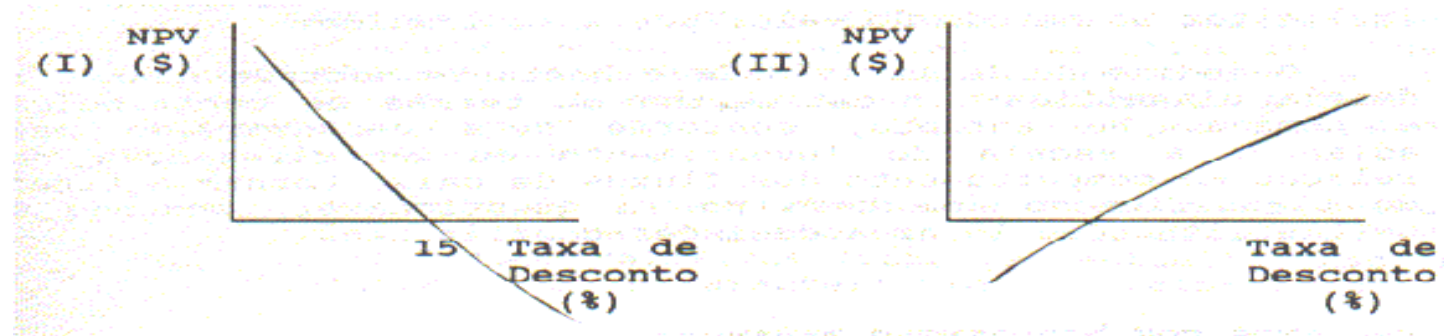

O g ráfico I é r epresentativo de um ap licador de capital que ap ura um a taxa de retorno de $15 \%$ ao período. O gráfico II fixa-se na posição de um tomador de capital que obtém emprestado recursos a um custo de $15 \%$ ao período.

A taxa int erna de retorno, de finida como a t axa de jur os que igua la o N PV a zero, é representada graficamente pe lo po nto q ue a linha do va lor $p$ resente lí quido $c$ orta o eix o d as ab scissas. Nas dua $s$ alternativas, IRR $=15 \%$.

No gráfico 1 o NPV d ecresce à m edida qu e a t axa de des conto s e elev a, apr esentando valores positivos até $15 \%$. Para esta taxa o NPV se an ula, in dicando a IRR da alt ernativa. Taxas maiores que $15 \%$ produzem um valor presente negativo, in dicando ser ec onomicamente de saconselhável a a Iternativa pa ra 0 aplicador.

Apesar do gráfico II ter sid o e laborado a partir do mesmo exemplo, os valores de caixa apresentam sinais invertidos, resultando em curva também inversa em relação ao gráfico 1. Esta taxa de $15 \%$ para quem toma c apital e mprestado é a t axa m ínima que deve ser auferida na aplicação desses recursos. Taxas de desconto m enores que $15 \%$ ao período produzem NPV negativo, e m aiores que 15\%, NPV positivo. Em conclusão, a IRR de $15 \%$ é a menor t axa de de sconto que produz um va lor presente liquid o po sitivo ao tomador do empréstimo. 
Os Métodos Quantitativos de Análise de Investimentos 12

\section{Índice de Lucratividade}

O índice de lucratividade (IL) é determinado pela divisão entre o valor presente dos benefícios de caixa e o valor presente dos desembolsos exigidos pelo investimento.

O IL, assim como as taxas de rentabilidade (TR), podem ser consideradas como variantes do NPV, proporcionando r esultados idên ticos em relação à s d ecisões de a ceitar-rejeitar alt ernativas de investimentos independentes. Apresentam ainda a $\mathrm{s}$ mesmas $\mathrm{v}$ antagens e $\mathrm{p}$ ressupostos im plícitos no $\mathrm{m}$ étodo do $\mathrm{v}$ alor presente líquido.

O índice de lucratividade é bastante adotado quando se deseja classificar investimentos em termos de contribuição econômica. No entanto, conforme será desenvolvido mais adiante, a escala do investimento ou as diferenças com relação ao comportamento dos fluxos de caixa tornam a tarefa de classificação com base no IL questionável, podendo em certas situações levar a decisões conflitantes.

\section{PROJETOS COM DIFERENTES TAMANHOS}

Resultados conflitantes com relação à aplicação dos métodos quantitativos de aná lise podem ocorrer quando as magnitudes dos investimentos (e também dos benefícios econômicos de ca ixa) se a presentarem desiguais.

Para ilus trar as ca racterísticas de sta sit uação, adm ita duas alt ernativas de investimento conforme identificadas a seguir. A taxa de retorno requerida para estes investimentos é de $20 \%$ ao período.

\section{PERÍODOS}

\begin{tabular}{llllllll} 
INV & O $123 \mathrm{~N}$ & \multicolumn{3}{l}{ PV IRR } & \multicolumn{1}{l}{ IL } \\
A & $\$ 450.000 \$ 3$ & $20.000 \$ 2$ & $30.000 \$ 1$ & $80.000 \$ 8$ & $0.555,60$ & $32,5 \%$ & 1,18 \\
B & $\$ 900.000 \$ 3$ & $60.000 \$ 2$ & $50.000 \$ 9$ & $00.000 \$ 9$ & $4.444,40$ & $25,6 \%$ & 1,10
\end{tabular}

Ao se considerar as duas alternativas de investimento como independentes (a decisão com relação a um investimento $\mathrm{n}$ ão a feta o o utro), $\mathrm{n}$ ão $\mathrm{h}$ á nenhum $\mathrm{c}$ onflito nos $\mathrm{r}$ esultados apurados. Tod os os métodos convergem para a atratividade econômica dos dois investimentos através do NPV positivo,

IRR maior que a taxa de retorno requerida e IL positivo e superior a 1,0 .

Não se verificando restrições de natureza técnica ou orçamentária, os dois projetos de investimento podem ser aceitos (implementados simultaneamente) como decorrência dos resultados positivos computados pelos métodos de análise.

Por outro lado, se os investimentos forem classificados como mutuamente excludentes, a es colha de uma alt ernativa e limina a possibilidade de se imple mentar outra, me smo que todas demonstrem atratividade econômica.

Avaliando os $r$ esultados $d$ a a nálise efetuada sobre os in vestimentos, evidencia-se uma situação decisorial de co nflito. P elo $m$ étodo do NPV, a alt ernativa B apr esenta-se $c$ omo $m$ ais a traente, se ndo classificada em primeiro lugar pelo maior montante esperado de riqueza. Inversamente, os métodos da IRR e do IL selecionam o investimento $\underline{A}$ como o mais atraente, proporcionando a melhor taxa percentual de retorno e lucratividade.

Esta dualidade de interpretação na seleção da melhor alternativa decorre em razão principalmente do método da IRR ser expresso em termos relativos ( taxa p ercentual) e $\mathrm{n}$ ão e $\mathrm{m}$ valores absolutos, como é característica do valor presente líquido.

Observe que o desembolso de capital de B é o dobro de A, e a IRR, por se apresentar referenciada em porcentagem, não leva em conta esta disparidade de tamanho. Em termos de riqueza absoluta, inerente ao método do NPV, é mais atraente apurar-se um resultado de $25,6 \%$ sobre $\$ 900.000,00$, do que de $32,5 \%$ sobre $\$ 450.000,00$.

Uma ou tra m aneira bastante es clarecedora de enfocar este pr oblema é e fetuar um a análise

Caderno de Estudos nº6, São Paulo, FIPECAFI - Outubro/1992 
Os Métodos Quantitativos de Análise de Investimentos 13

incremental do $s$ investimentos. $A$ dif erença en tre os projetos $B$ e $A$ é qu e $B$ exige um inv estimento de $\$ 450.000,00 \mathrm{~m}$ aior, prometendo e $\mathrm{m}$ co nseqüência fluxos de c aixa ad icionais de $\$ 4 \quad 0.000,00, \$ 20.000,00 \mathrm{e}$ $\$ 720.000,00$, respectivamente, ao final dos próximos três períodos, isto é:

\begin{tabular}{|c|c|c|c|c|}
\hline \multicolumn{5}{|c|}{ PERÍODOS } \\
\hline & $\mathrm{O}$ & 1 & & 3 \\
\hline INVEST.B: & $-\$ 900.000,00$ & $\$ 360.000,00$ & $\$ 250.000,00$ & $\$ 900.000,00$ \\
\hline INVEST.A: & $-\$ 450.000,00$ & $\$ 320.000,00$ & $\$ 230.000,00$ & $\$ 180.000,00$ \\
\hline $\begin{array}{l}\text { VALORES IN } \\
\text { CREMENTAIS- }\end{array}$ & $\$ 450.000,00$ & $\$ 40.000,00$ & $\$ 20.000,00$ & $\$ 720.000,00(B-A)$ \\
\hline
\end{tabular}

Apurando-se o valor presente liquido e a taxa interna de retorno do investimento incremental, chega-se aos seguintes resultados positivos em termos de atratividade dos investimentos:

* $\quad \Delta \mathrm{NPV}=\$ 13.888,90$ (valor presente líquido incremental)

* $\Delta \mathrm{IRR}=\quad 21,3 \%$ ao período (taxa interna de retorno incremental).

O N PV in cremental def ine a $r$ iqueza a dicional ac rescida p elo inv estimento $\underline{B}$ de ma ior e scala. $E \mathrm{~m}$ outras palavras, é o custo máximo a que o investimento B pode se elevar para que mantenha a sua preferência em relação a $A$.

A IRR representa a taxa de jur os qu e t orna os $d$ ois inv estimentos e quivalentes e $m t$ ermos de atratividade econômica, produzindo o mesmo valor presente líquido. Esta taxa é reconhecida por intercessão de $\mathrm{F}$ isher. Para uma taxa de des conto de até $21,3 \%$ ao pe ríodo, o inv estimento $\mathrm{B}$ é $\mathrm{p}$ referível a $\mathrm{A}$, apresentando maior riqueza líquida. A partir de 21,3\%, no ent anto, o in vestimento A pa ssa a s er o mais atraente.

Em termos gráficos, tem-se o seguinte comportamento dos investimentos A e B:

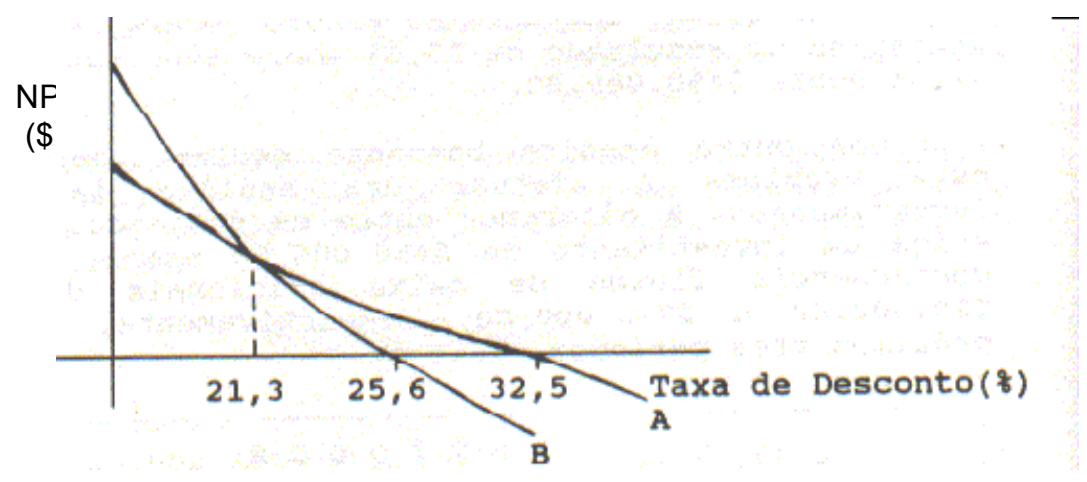

Como a taxa mínima de atratividade para os investimentos é de $20 \%$ ao período, a alternativa B é a que p romove, $\mathrm{p}$ ara es ta taxa de de sconto, o m aior va lor presente lí quido, s endo, po rtanto, a $\mathrm{m}$ elhor opção econômica de investimento.

Na situação descrita de conflito decisorial em projetos com disparidade de tamanho, o método do NPV é aceito como o que produz as melhores recomendações. A aplicação do IRR identifica algumas dificuldades em relação à seleção das alternativas, pois o método não leva em conta a escala do investimento. 
Os Métodos Quantitativos de Análise de Investimentos 14

\section{NPV e IL Aplicados em Investimentos Mutuamente Excludentes} do NPV.

Foi observado anteriormente que o índice de lucratividade produz as mesmas conclusões do m étodo

$\mathrm{Na}$ análise de investimentos independentes, o uso do IL é simples. Aceita-se todo projeto que apresenta índice superior a 1,0, o que indica um NPV maior que zero.

Em alternativas mutuamente excludentes, no entanto, a escolha do investimento com maior índice de lucratividade pode não refletir a melhor decisão. As razões são as mesmas apontadas anteriormente para o método da IRR: disparidade de tamanho dos investimentos e diferenças com relação à evolução dos fluxos de caixa ao longo do tempo.

$\mathrm{Na}$ ilustração desenvolvida anteriormente, observa-se que as indicações de seleção dos métodos não são coincidentes. Pelo NPV escolhe-se o investimento B, e pelo IL a alternativa A é a selecionada.

Uma vez mais a falha é verificada no fato do método não levar em consideração a escala do investimento, como é efetuado pelo NPV. Na análise incremental realizada, tem-se o seguinte índice:

AIL $=\$ 463.888,90 / \$ 450.000,00=1,03$

O índice de lucratividade incremental é superior a 1,0, indicando a atratividade do investimento.

A melhor decisão pode ser mais facilmente tomada selecionando-se a alternativa com maior valor presente liquido. A análise incremental corrobora a recomendação do método do valor presente líquido.

Por outro lado, em situações que envolvem investimentos com disparidade mas que produzem o mesmo valor presente líquido, a orientação de superioridade do método do NPV pode ser questionada.

Para ilustrar esta situação, são apresentados a seguir os investimentos $\underline{C}$ e $D$, para os quais está definida uma taxa mínima de atratividade de $20 \%$ ao período.

Pelo método do NP V os dois inv estimentos são atraentes e economicamente equivalentes, pois produzem o mesmo resultado líquido no momento presente.

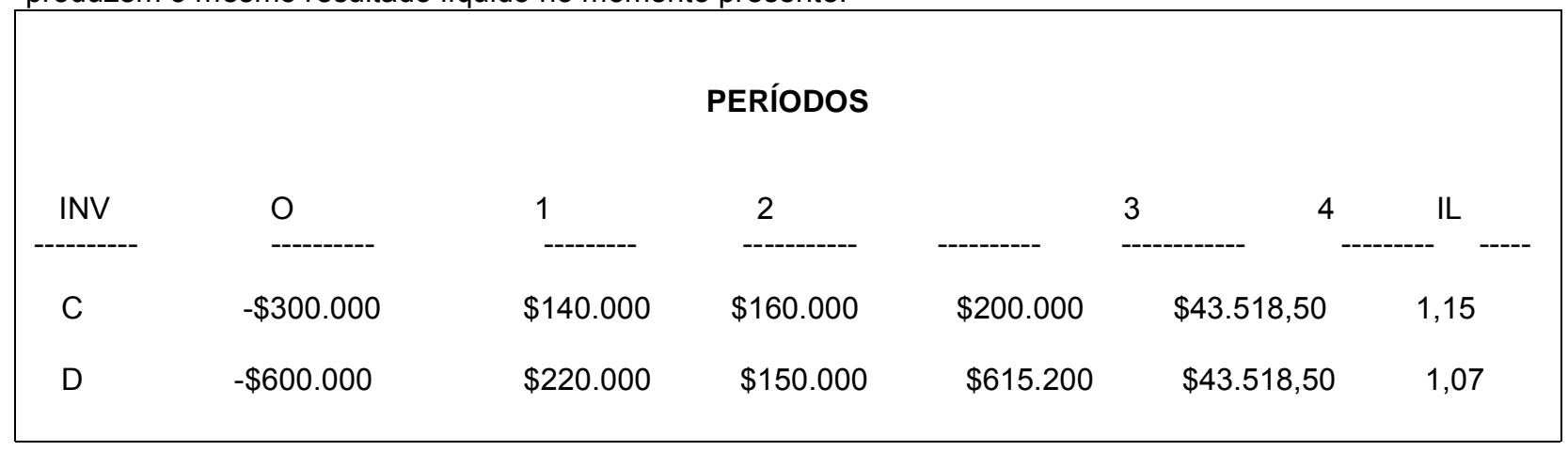

A c omparação envolve do is investimentos com dif erentes $t$ amanhos (o inv estimento $D$ ex ige um desembolso de capital duas vezes maior que ) e que produzem o mesmo valor presente líquido.

Em condições de restrição de c apital é necessário levar-se em c onta a relação do va lor pr esente líquido com o volume de recursos demandado pelo investimento, de forma a a purar-se o retorno of erecido por cada unidade de capital aplicado.

$O$ índice de luc ratividade considera este aspecto essencial à análise, oferendo outros indicativos para a decisão. Para um mesmo NPV, obtém - se uma taxa de retorno maior na implementação do investimento C $(I L=1,15)$ do que em $D(I L=1,07)$.

\section{PROJETOS SEM INVESTIMENTO INCREMENTAL}

Em algumas situações de seleção de investimentos pode se deparar com alternativas apresentando diferentes e conflitantes r esultados econômicos, $\mathrm{m}$ as demandando o mesmo va lor de desembolso inicial. Nestes casos não há investimento incremental, conforme foi discutido ao se tratar de projetos com disparidade 
de tamanho. A análise é efetuada sobre o comportamento dos fluxos de caixa ao longo do tempo.

Considere ilustrativamente os investimentos E e F descritos a seguir. A taxa de atratividade é fixada em $20 \%$ ao período.

\begin{tabular}{|c|c|c|c|c|c|}
\hline & & & PERÍODOS & & \\
\hline INV & $\mathrm{O}$ & 1 & 2 & NPV & IRR \\
\hline$E$ & $-\$ 500.000,00$ & $\$ 650.000,00$ & $\$ 100.000,00$ & $\$ 111.111,10$ & $43,0 \%$ \\
\hline $\mathrm{F}$ & $-\$ 500.000,00$ & $\$ 80.000,00$ & $\$ 820.000,00$ & $\$ 136.111,10$ & $36,3 \%$ \\
\hline
\end{tabular}

Admitindo-se inic ialmente que os investimentos sejam independentes, is to é, podem s er implementados simultaneamente, a o rientação dos resultados dos métodos de a nálise é pe la ac eitação da s duas $p$ ropostas. A presentam N PV po sitivo e a I RR d os inv estimentos supera a t axa m ínima de retorno requerida.

No entanto, ao se considerar os investimentos como mutuamente excludentes, surge uma divergência técnica de or ientação, O m étodo do N PV s eleciona o investimento $\mathrm{F}$ c omo o m ais at raente (maior $r$ iqueza absoluta), e o método da IRR indica E como a mais desejável (maior taxa percentual de retorno).

Os inv estimentos ap resentam alg umas c aracterísticas que os dif erenciam da s ituação ant erior co $\mathrm{m}$ distintas e scalas. Os d ois projetos demandam o mesmo volume de des embolso inic ial $(\$ 500.000,00)$, $\mathrm{m}$ as apresentam nítidas diferenças no perfil de formação de seus benefícios de caixa ao longo do tempo.

No projeto $E$, os fluxos de caixa comportam-se de maneira decrescente no tempo, e no projeto $F$, de forma oposta, os fluxos de caixa são crescentes.

Essa dualidade de comportamento explica a natureza d o conflito pr oporcionada $p$ elo $m$ étodo de análise no tocante à s eleção da m elhor alt ernativa de inv estimento. O s métodos qu antitativos de análise trazem implícito o pressuposto de reinvestimento dos fluxos de caixa pela taxa de desconto utilizada (método do NPV) ou pela própria taxa de retorno calculada (método da IRR).

Nestas condições d e $r$ einvestimento au tomático, o $\mathrm{m}$ étodo qu e a presentar $\mathrm{f}$ luxos de ca ixa decrescentes ( valores maiores no iní cio) é levado a det erminar a maior IRR. E m verdade, quanto mais elevados se apresentarem os fluxos de caixa nos momentos iniciais do investimento, maior é a IRR calculada, uma vez que se assume que os valores de caixa são reinvestidos a esta taxa de juros.

O mesmo não se verifica com o método do NPV. O método admite reinvestimento à taxa de desconto utilizada, geralmente a IRR calculada. Fluxos de caixa mais elevados em períodos mais distantes promovem maior valor presente quando descontados pela taxa mínima de atratividade do que quando adotada a taxa interna de retorno.

Pela intercessão de Fisher, identificada pela taxa interna de retorno do investimento incremental (F E), chega-se à taxa de juros de indiferença de $26,3 \%$ ao período, ou seja:

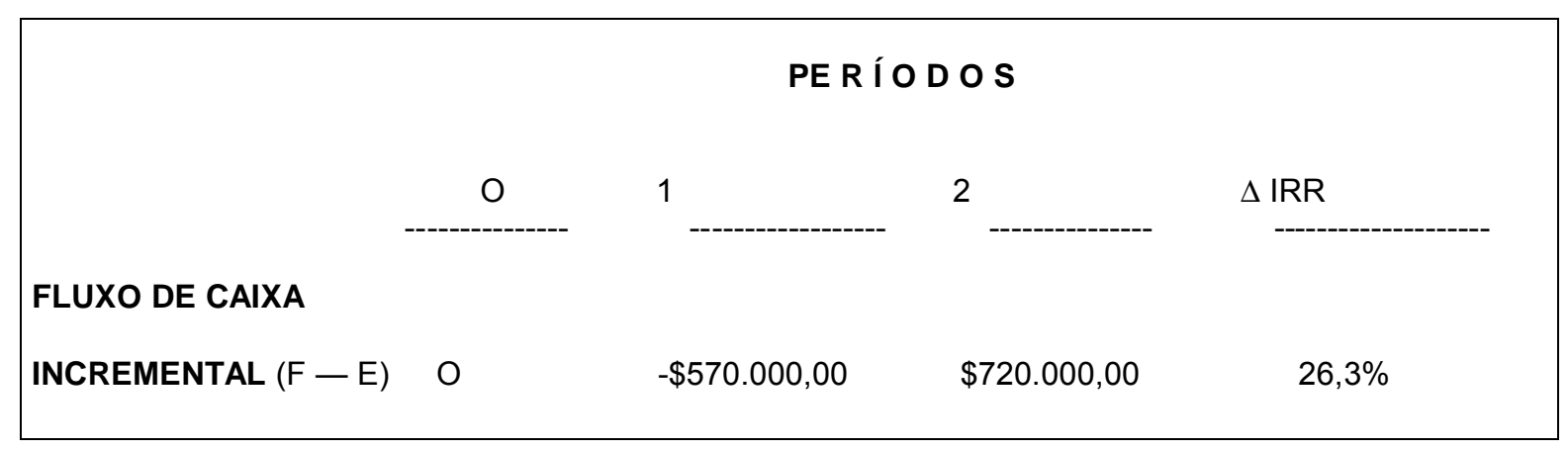

Graficamente, tem-se a seguinte representação:

Caderno de Estudos nº6, São Paulo, FIPECAFI - Outubro/1992 
Os Métodos Quantitativos de Análise de Investimentos 16

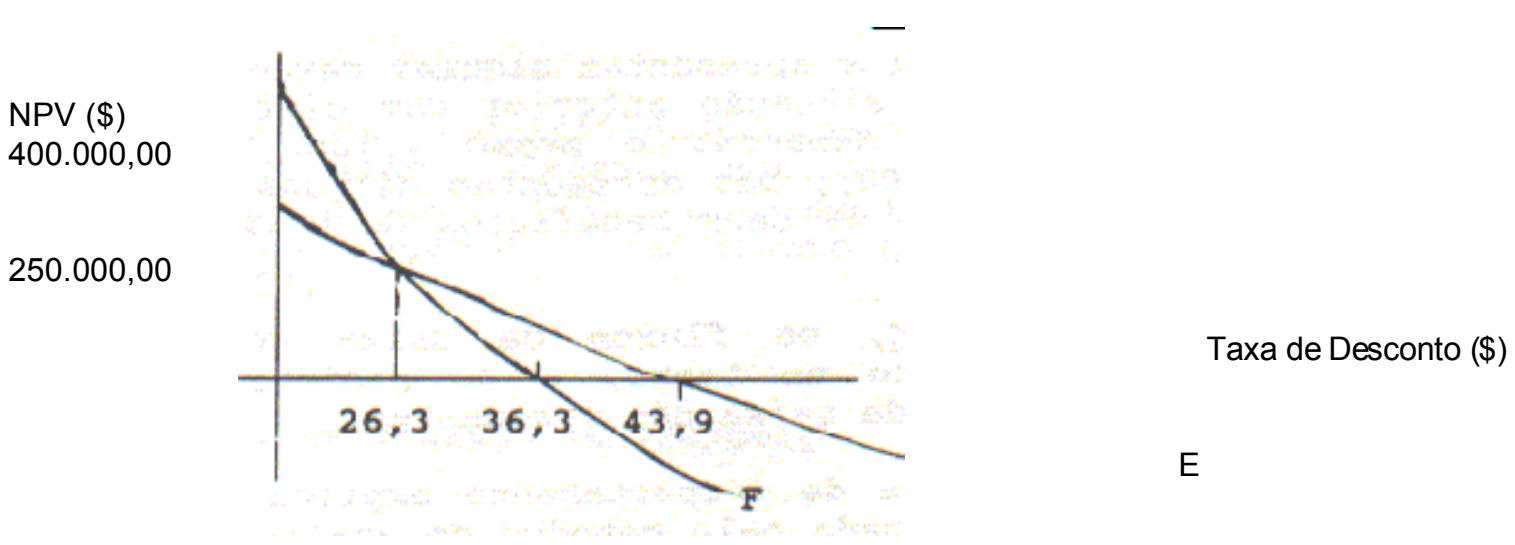

Até a taxa de investimento de $26,3 \%$ (ponto de indiferença), o projeto $\mathrm{F}$ é o mais desejável, apurando maior valor presente liquido. A partir desta taxa até $43,9 \%$, o investimento $E$ passa a ser o mais atraente.

Sendo de $20 \%$ ao período a taxa de at ratividade para as propostas, o pr ojeto , de $\mathrm{m}$ aior N PV, destaca-se como o mais desejável. No raciocínio da decisão, admite-se como mais provável o reinvestimento dos fluxos de caixa à taxa de retorno requerida do que a IRR calculada. Observe que, uma vez mais, a análise se concreta na taxa de reinvestimento dos fluxos de caixa.

\section{CONCLUSÕES}

O trabalho teve o objetivo de evidenciar os principais aspectos críticos dos métodos quantitativos de análise de investimentos. Foram considerados no estudo os métodos do fluxo de caixa descontado mais utilizados pelas empresas: valor presente líquido, taxa interna de retorno e índice de lucratividade.

Pela sua grande simplicidade e ampla utilização prática, foram também efetuadas algumas discussões sobre o período de "payback". Diante das limitações técnicas bastante conhecidas, foi proposto que o seu uso fosse feito em valores de caixa atualizados.

Para os métodos de fluxo de caixa descontado, tecnicamente mais corretos, prevalece, na maioria dos casos, a superioridade do valor presente líquido. No entanto foi discutida uma situação em que o índice de lucratividade produz informações mais completas, principalmente diante de restrições orçamentárias.

\section{BIBLIOGRAFIA}

a) ASSAF NETO, Alexandre \& MARTINS, Eliseu. Administração Financeira. São Paulo, Atlas, 1.985.

b) ABREU, Paulo F. Simas P. de \& STEPHAN, Christian. Análise de Investimentos. Rio de Janeiro, Editora Campus, 1.982.

c) BIERMAN, JR. Harold \& SMIDT, Seymour. The Capital Budgeting Decision. McMillan Publishing, 4' ed.

d) DRIMER, R oberto L. " Criterios $C$ uantitativos Para La Evaluación de Pr oyectos'. Bu enos Aires, Administración de Empresas, 1.988, Tomo XIX. 